\title{
ANN based Day-Ahead Spinning Reserve Forecast for Electricity Market Simulation
}

\author{
Pedro Faria, Zita A. Vale, IEEE Member, João Soares, Hussein Khodr, IEEE Member
}

\begin{abstract}
Electricity market players operating in a liberalized environment require adequate decision support tools, allowing them to consider all the business opportunities and take strategic decisions. Ancillary services represent a good negotiation opportunity that must be considered by market players. This paper deals with short-term predication of day-ahead spinning reserve (SR) requirement that helps the ISO to make effective and timely decisions. Based on these forecasted information, market participants can use strategic bidding for day-ahead SR market. The proposed concepts and methodologies are implemented in MASCEM, a multi-agent based electricity market simulator. A case study based on California ISO (CAISO) data is included; the forecasted results are presented and compared with CAISO published forecast.
\end{abstract}

Index Terms-Artificial neural networks (ANN), ancillary services, multi-agent systems, spinning reserve, electricity markets, power systems, simulation

\section{INTRODUCTION}

$\mathrm{C}$ ONTROLLING frequency and voltage has always been an essential part of operating a power system. In the scope of the liberalization of electricity markets, the resources required to achieve this control should be treated as services that the system operator can acquire from other market players.

Ancillary services (AS) are essential for secure, stable and economical operation of the power system, playing a vital role in free and fair electricity trade in competitive power markets. The AS procurement is a major operational function for the Independent System Operator (ISO).

Spinning Reserve (SR) is one of the most important AS required for maintaining power system reliability following a major contingency. Short-term predication of day-ahead SR requirement helps the ISO to make effective and timely decisions. Moreover, based on these forecasted information, market participants can derive the optimal bidding strategies for day-ahead SR market.

As electricity market liberalization has been achieved by independent evolution processes in different parts of the

The authors would like to acknowledge FCT, FEDER, POCTI, POSI, POCI, POSC and POTDC for their support to R\&D Projects and GECAD Unit.

P. Faria, Z. Vale, J. Soares and H. Khodr are with GECAD Knowledge Engineering and Decision-Support Research Center of the Electrical Engineering Institute of Porto - Polytechnic Institute of Porto (ISEP/IPP), Rua Dr. António Bernardino de Almeida, 431, 4200-072 Porto, Portugal (e-mail: pnf@isep.ipp.pt ; zav@isep.ipp.pt , jxs@isep.ipp.pt, hmk@isep.ipp.pt ). world and because of the structural differences in the underlying power systems, the technical definitions of these services and the rules that govern their trading are significantly different from market to market. In many countries, the ISO has the overall responsibility of providing and procuring the required AS on behalf of market participants, which do not self provide these services, through a competitive bidding process. The ISO's objective is to minimize AS costs while encouraging participants to provide the required AS. On the other side, energy producers can use strategic bidding in order to maximize their profits [1].

This paper presents an ANN approach for day-ahead SR forecasting which has been included in MASCEM. MASCEM [2] is a multi-agent based electricity market simulator that uses sophisticated Artificial Intelligence based techniques for players modeling and decisionsupport. It uses a flexible and open approach that supports diversity of electricity market (EM) models and of tools to be used by market players, and a balanced approach of technical and economic issues.

MASCEM has been designed to simulate the energy market, supporting a pool and bi-lateral contracts. Recently it has been upgraded to support the modeling and simulation of aggregators. Virtual Power Plants (VPP) [3] are an important type of aggregator that is especially relevant to aggregated distributed generation, heavily based on renewable-based sources, in order to overcome their weak points and make them valuable in a competitive market.

The simulation studies that have been undertaken show that the aggregation of such producers allows them to gain competitive advantage and are a very interesting mean for them to participate in the market. However, the results already obtained show that these players' strategies must consider all the available business opportunities and not only the energy market.

Only a tool able to simulate all the possible negotiation opportunities is of value for electricity market players and regulators as a partial view does not allow to experiment and train negotiation strategies in a realistic way. Derivative market is important as is allows to practice the hedge and undertake strategic risk management. Ancillary services can be used by producers to increase their income. For this reason, methods for portfolio optimization [4] and reserve management [5] have been developed and tested.

In order that MASCEM can provide electricity market players with a realistic and useful simulation platform, if must include mechanisms to support ancillary services (AS) provision. This paper deals with short-term predication of 
day-ahead spinning reserve (SR) requirements using an ANN based approach. The proposed concepts and methodologies are implemented in MASCEM. A case study based on California ISO (CAISO) data is included; the forecasted results are presented and compared with CAISO published forecast.

\section{ARTIFICIAL NEURAL NETWORKS}

Neural networks can learn to approximate any function and behave like associative memories using sample input/output data that is representative of the desired task, operating then as are model free estimators.

This gives them a key advantage over traditional approaches to function estimation such as the statistical methods used in adaptive control. Neural systems estimate a function without requiring a mathematical model of how the output functionally depends on the input.

ANNs consist of an organized set of neurons that can be modified to realize a desired behavior. In summary, they learn to approximate sampled functions even when their form cannot be specified precisely. ANN models represent an approach to systems engineering that is potentially robust and fault tolerant.

\section{A. General Remarks}

Association, optimization, feature extraction and recognition operations can be performed using artificial neural networks. In such applications, neural networks can learn from many operating conditions and thus have the ability to make the decisions needed to identify whether changes are significant.

A neural network has its neurons linked by connections that can be strengthened or weakened, this concept being based on the interconnected neurons of the brain. Neural networks are "trained" by being given a series of examples of correct responses; the connections between neurons are strengthened or weakened according to the level of success in reproducing what is desired. The network is never given an explicit body of rules to follow: the strengths and weaknesses of the different links within it define its course. Fig.1 illustrates a typical ANN neuron.

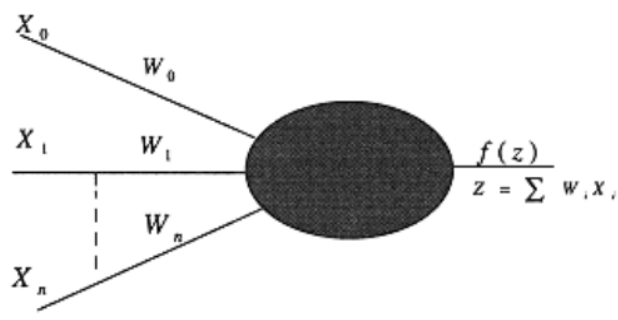

Fig. 1. Typical neuron.

Since a single "hidden" layer (a layer of nodes between the input and output-layer) is usually adequate to approximate any continuous function, it follows that the same property is also enjoyed by networks with more than one hidden layer. Although the knowledge about neural network design do not provide a recipe for the choice of the number of layers and the number of nodes in each layer. This is verified, manly, in the case of function approximation techniques with functions like polynomials, Fourier series and general orthogonal functions [6].

\section{B. Multi-Layer Networks}

The most widely used artificial neural paradigm is probably the Multi-Layer Perceptron (MLP). The typical neuron presented in Fig. 2 shows the processing of the input vector. A larger network structure is produced by interconnecting many neurons.

The output of the network is attained by forming the dot product of an input and a weight vector, and then nonlinearly transforming this variable using a bounded, continuous non-linear function such as the S-shaped sigmoidal function.

The sigmoid $f(z)$ transfer function (1) takes the input (which may have any value between $+\infty$ and $-\infty$ ) and maps the output into the range $[0,1]$. The sigmoid function $f(z)$ is mathematically defined as:

$f(z)=\frac{1}{e^{-z}} \in(0,1)$

where the input $z=X T W$. This function is extensively used in Back Propagation (BP) networks mainly due to the fact that $f(z)$ is differentiable. This operation can be distributed across several layers, where the output of one layer forms the input to another, and the magnitude and orientation of the weight vectors determine how knowledge is distributed in the overall network [7]. An MLP network contains the universal approximation property, and it is available in many software packages and on specific microprocessors. These properties, coupled with the fact that the simple instantaneous gradient descent BP learning rule can be used to train the weights, mean that this learning paradigm has attracted the attention of many engineers in recent years.

\section{Radial Basis Function (RBF) Network}

The Radial Basis function (RBF) network consists of one hidden layer of basis functions, or neurons. At the input of each neuron, the distance between the neuron center and the input vector is calculated. A distance measure, to determine how far an input vector is from the centre, is typically the standard Euclidean distance measure. The node calculates the Euclidean distance between the centre and the input vector of the network and the result is passed through a non-linear function. The output of the neuron is then formed by applying the basis function to this distance. The $\mathrm{RBF}$ network output is formed by a weighted sum of the neuron outputs and the unity bias shown.

\section{ANN for AS Forecasting}

The ISO and GENCOs need a reasonable procedure to forecast the SR required in day-ahead competitive electricity markets. ANNs have gained significant attention 
to be used for this purpose because of their clear model, easy implementation, and good performance.

This paper proposes an ANN based methodology for spinning reserve forecasting which has been implemented using MATLAB. The whole process consists of two stages: the training stage and the forecasting stage. Each stage is an adaptive process in the sense that it includes a feedback process that allows the artificial neural network to learn from its mistakes and correct its output by adjusting its neurons.

At the training stage, the proper training matrix is identified; the number and the type of neurons and layers defines the precision and computation resources for spinning reserve probability forecasting. The complexity of the training stage depends on the type of application that is proposed (marketing, generation, etc.). The training stage can end being a cumbersome process if it is not done in the right way. For instance, the overtraining of neurons can seriously deteriorate the forecasting results. Furthermore, training the ANNs based on a training matrix that is very different from the input matrix can also make the forecasting useless.

\section{ANCILlary SERVICES MARKETS}

Electricity markets have as the underlying negotiated product the electrical energy required by the demand side; the energy transactions occur in the scope of the energy market. Additionally, services required for assuring security and reliability, known as ancillary services (AS), must be obtained [8-13]. Ancillary services include active power AS (load/frequency control (LFC), including primary control, automatic generation control (AGC), tertiary control, balancing service and black start provision) and reactive power AS (voltage/reactive power control).

Ancillary services are the services that are important to achieve targeted objectives on power system security, frequency stability, as well as voltage level and stability. Operating reserve is an important commodity in the category of ancillary services, which supports the system reliability against unexpected generators outages.

In electricity markets AS can be seen as a set of products separated from the energy production. A competitive environment requires adequate procedures and methodologies for determining AS needs, the ways they are obtained and priced. These must assure that the AS required for assuring PS security and reliability are permanently available and these services are fairly remunerated. AS provision can be mandatory or remunerated under marketdriven mechanisms. AS can be treated jointly with the energy market or on a separated basis; a hybrid approach, treating a part of AS (e.g. frequency control) jointly with the energy markets and the remaining AS separately is also possible. As with any commodity, creating a market for ancillary services is a way of resolving the conflict between buyers and sellers to their mutual benefit. Transmission system operators (TSOs) objective is to obtain the resources they need to maintain the security of the system at minimum cost, while providers aim at maximizing their profits. However, ancillary services are not simple commodities and have strong requirements for assuring power system security. Due to this, a diversity of AS trading methods is used around the world. MASCEM aims at modeling the most relevant methodologies used for AS trading so that it can provide market players with adequate decision support.

\section{A. CAISO}

In California electricity market, the energy market and the ancillary service markets are separately managed by two different entities, i.e., the Power Exchange (PX) and the ISO respectively. To ensure compliance with Western Electricity Coordinating Council's (WECC) Minimum Operating Reliability Criteria (MORC), CAISO procures following AS requirements on an hourly basis in both the day-ahead and hour-ahead markets in a sequential manner [14].

The CAISO operates a separate market for each AS. Schedule Coordinators (SCs) that wish to provide AS to CAISO may either submit AS bids or self-provide AS.

Submitted AS bids must contain a capacity component and an energy component. The selection of AS suppliers is solely based on the capacity bids and the required AS amount broadcast by the CAISO. Acceptance of selfprovided AS occurs prior to AS bid evaluation in the relevant market and effectively reduces the AS requirements that need to be met by AS bids within the same AS region, and reduces the AS obligation for the SC self-providing the AS, in the AS cost allocation.

\section{CASE STUDY}

This section presents an example of the application of the proposed methodology to CAISO data.

\section{A. California Electricity Market Data}

This case study considers day-ahead hourly final SR requirements of CAISO grid for the 2007 winter season. Relevant data have been obtained from CAISO website [14].

CAISO day-ahead hourly SR requirement and load data of 48 days before the day whose SR requirements are to be forecasted are used to build the forecasting model. The last week of winter season, that corresponds to the last week of February (from Feb. 19th to Feb. 25th) has been used to test the performance of the proposed ANN. The hourly data used in the present study correspond to the period from January 2nd to Feb. 18th, 2007.

Reference [13] clearly shows that SR requirements are highly correlated with the FD and therefore it is used as an exogenous variable to predict the SR requirements of the CAISO controlled grid. It is clear, too, that the day-ahead hourly AS requirement series exhibits multiple seasonal patterns corresponding to daily and weekly seasonalities. The required reserve of 3 hours before the hour to be predicted $S R_{h-3}$ is highly correlated with the requirements for the hour to be predicted too. This information is, therefore, included in the input layer data.

The present paper proposes a prediction of SR with two alternative approaches - with or without FD historical data - called With Load and Without Load respectively. In [13], 
an Adaptive Wavelet Neural Network (AWNN) is employed to do the same prediction. AWNN is a Wavelet Neural Network that employed wavelet activation functions from continuous wavelet transform.

The Cross Correlation Function (XCF) between hourly $\mathrm{SR}$ requirements and the load was useful to decide the number of lagged values of CAISO load to be used to predict the SR requirements. With consideration of all the discussed factors, the set of input variables used to forecast the spinning reserve $S R_{h}$ at hour h, is the exposed in (2) for the approach including all data (SR and load data) and the exposed in (3) for the approach that does not consider load data.

The structure of the RBF network, with an output vector and an input vector, is shown in Fig.2 considering load.

$$
\left\{\begin{array}{l}
S R_{\mathrm{h}-1}, S R_{\mathrm{h}-2}, S R_{\mathrm{h}-3}, S R_{\mathrm{h}-23}, S R_{\mathrm{h}-24}, S R_{\mathrm{h}-25}, S R_{\mathrm{h}-48}, \\
S R_{\mathrm{h}-72}, S R_{\mathrm{h}-96}, S R_{\mathrm{h}-120}, S R_{\mathrm{h}-144}, S R_{\mathrm{h}-168}, S R_{\mathrm{h}-169}, S R_{\mathrm{h}-192}, \\
F D_{\mathrm{h}-1}, F D_{\mathrm{h}-2}, F D_{\mathrm{h}-3}, F D_{\mathrm{h}-24}, F D_{\mathrm{h}-48}, F D_{\mathrm{h}-72}, \\
F D_{\mathrm{h}-96}, F D_{\mathrm{h}-120}, F D_{\mathrm{h}-144}, F D_{\mathrm{h}-168}
\end{array}\right\}
$$

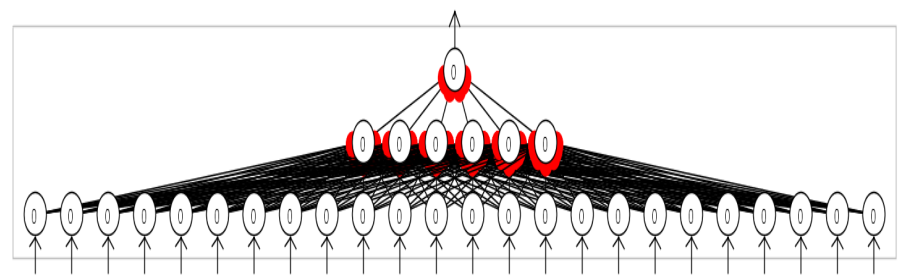

Fig. 2. RBF Network structure - With Load approach.

Equation (3) and Fig.3 correspond to the RBF approach when the load is not considered.

$$
\left\{\begin{array}{l}
S R_{\mathrm{h}-1}, S R_{\mathrm{h}-2}, S R_{\mathrm{h}-3}, S R_{\mathrm{h}-23}, S R_{\mathrm{h}-24}, S R_{\mathrm{h}-25}, S R_{\mathrm{h}-48}, \\
S R_{\mathrm{h}-72}, S R_{\mathrm{h}-96}, S R_{\mathrm{h}-120}, S R_{\mathrm{h}-144}, S R_{\mathrm{h}-168}, S R_{\mathrm{h}-169}, S R_{\mathrm{h}-192},
\end{array}\right\}
$$

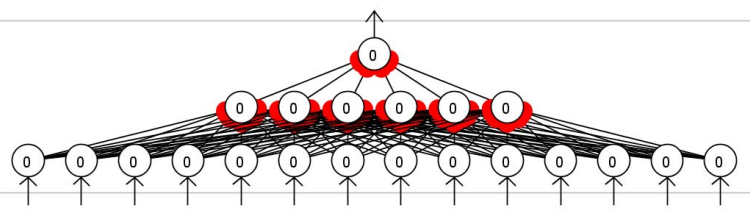

Fig. 3. RBF Network structure - Without Load approach.

The historical data collected in CAISO website includes values of load and SR to the 48 days before the test week plus the test week values, corresponding to a total of 1320 periods $(=24 * 48+24 * 7=1152+168)$. Both approaches need the data of 192 hours ago to predict the SR requirements for hour h, therefore $960(=1152-192)$ input vectors are used to train the network.

Table I shows, for each approach, the number of neurons in the input layer, the number of hidden layers and the number of neurons in these, and the learning rate. All models have one neuron in the output layer (the SR requirement for hour $\mathrm{h}$ ).
TABLE I

NETWORK STRUCTURE AND DATA

\begin{tabular}{|c|c|c|c|}
\hline APPROACH & INPUT & $\begin{array}{c}\text { HIDDEN } \\
\text { LAYER }\end{array}$ & LEARNING RATE \\
\hline$[13]$ & 24 NEURONS & $\begin{array}{c}\text { 1 LAYER WITH } \\
\text { 3 NEURONS }\end{array}$ & 0.1 \\
\hline WITH LOAD & 24 NEURONS & $\begin{array}{c}1 \text { LAYER WITH } \\
6 \text { NEURONS }\end{array}$ & 0.1 \\
\hline WITHOUT LOAD & 14 NEURONS & $\begin{array}{c}\text { 1 LAYER WITH } \\
6 \text { NEURONS }\end{array}$ & 0.1 \\
\hline
\end{tabular}

In the proposed approaches, a structure with 1 hidden layer with 6 neurons was selected after a large number of trials.

\section{B. SR Forecasting Results}

The performance of the forecasting models in terms of daily and weekly Mean Absolute Percentage Error (MAPE) and Weekly error variance were calculated. DMAPE (4) and WMAPE (5) are acronyms for daily MAPE and weekly MAPE respectively. Smaller values of WMAPE mean predicted values closer to the real values. DMAPE and WMAPE are calculated according to (4) and (5) respectively:

$$
\begin{aligned}
& D M A P E=\frac{1}{24} \sum_{h=1}^{24} \frac{\left|S R_{h}^{a c t}-S R_{h}^{\text {for }}\right|}{S R_{h}^{a c t}} \times 100 \\
& W M A P E=\frac{1}{168} \sum_{h=1}^{168} \frac{\left|S R_{h}^{a c t}-S R_{h}^{f o r}\right|}{S R_{h}^{a c t}} \times 100
\end{aligned}
$$

where $\mathrm{h}$ is the hour for which the forecast is being undertaken, and $S R_{h}^{a c t}$ and $S R_{h}^{\text {for }}$ are the actual and forecasted values of the SR, respectively.

Table II presents the day-ahead hourly SR requirements forecast performance indexes for $\mathrm{ANN}, \mathrm{AWNN}$, and CAISO, obtained from [13] and [14], and of our proposed ANN based methodology with and without load forecast for

\begin{tabular}{|c|c|c|c|c|c|}
\hline MODELS & $\begin{array}{c}\text { CAISO } \\
{[14]}\end{array}$ & $\begin{array}{c}\text { ANN } \\
{[13]}\end{array}$ & $\begin{array}{c}\text { AWNN } \\
{[13]}\end{array}$ & $\begin{array}{c}\text { PROPOSED } \\
\text { ANN } \\
\text { WITH } \\
\text { LOAD }\end{array}$ & $\begin{array}{c}\text { PROPOSED } \\
\text { ANN } \\
\text { WITHOUT } \\
\text { LOAD }\end{array}$ \\
\hline WMAPE & 5.952 & 4.106 & 3.710 & 3.674 & 3.729 \\
\hline
\end{tabular}
the considered test week.

TABLE II

WMAPE COMPARATIVE RESULTS

The AWNN methodology proposed in [13] had a run time of five minutes using MATLAB 7.1 using a PC with 512MB RAM and $3.0 \mathrm{GHz}$ Pentium 4 processor. The ANN methodology proposed in this work has been implemented using MATLAB 7.6 and run in a based $3.4 \mathrm{GHz}$ Pentium 4 PC with 1GB RAM computer in 30 seconds.

It can be observed that weekly MAPE values for AWNN model are lower than those of ANN model and CAISO published hourly SR requirements forecast results. The evaluation of the forecasted values obtained with our methodology, considering load values, shows that the results are between those of ANN and AWNN.

For a better insight of the forecasted results, the daily MAPE with and without load for the results obtained with 
the proposed methodology are shown in Fig. 4. DMAPE values for AWNN, ANN and CAISO results are also shown in Fig. 4.

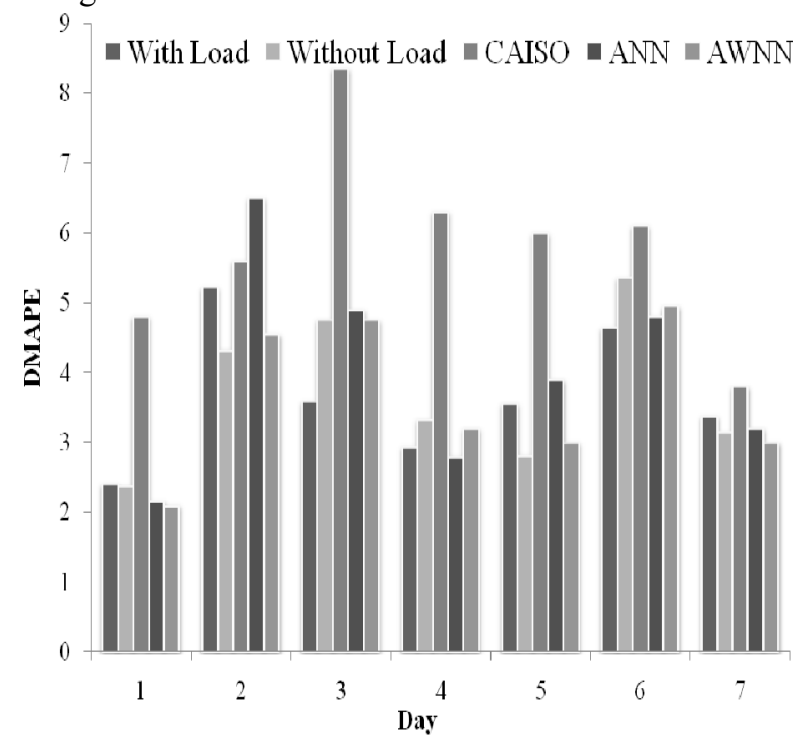

Fig. 4. Daily MAPE results for test week.

Fig. 5 shows the results obtained published in [13]. Fig.6 and Fig.7 show the forecasted results along with actual values for the winter test week, using the proposed ANN model and CAISO published forecast results. These results clearly show that ANN with load data predicts the irregular spikes of day-ahead hourly SR requirements better in comparison to ANN without load data.

In Fig.5, Fig.6, and Fig.7 actual values correspond to the real values that occurred on CAISO market.

Moreover, since the CAISO published hourly SR requirements forecast results are based on the CAISO load forecast, the forecast accuracy of hourly SR requirements depends on how accurate the load forecast is. Therefore, CAISO forecast results are not much accurate particularly for irregular spikes of hourly SR requirements.

\section{CONCLUSIONS}

Providing adequate decision support to electricity market players requires the use of decision support tools which can be effectively based on simulation studies. In order to make decision support effective, all business opportunities must be considered. For this reason, the authors have included derivative market and ancillary market models in MASCEM, a multi-agent electricity market simulator.

This paper presents an ANN based methodology for spinning reserve forecast which has been implemented in MATLAB and integrated with MASCEM. The use of the proposed methodology for real data demonstrates that it can be used in realistic simulations. The results of these simulations are later used as historic data for learning purposes, resulting in the suggestion of adequate bidding strategies for producers.

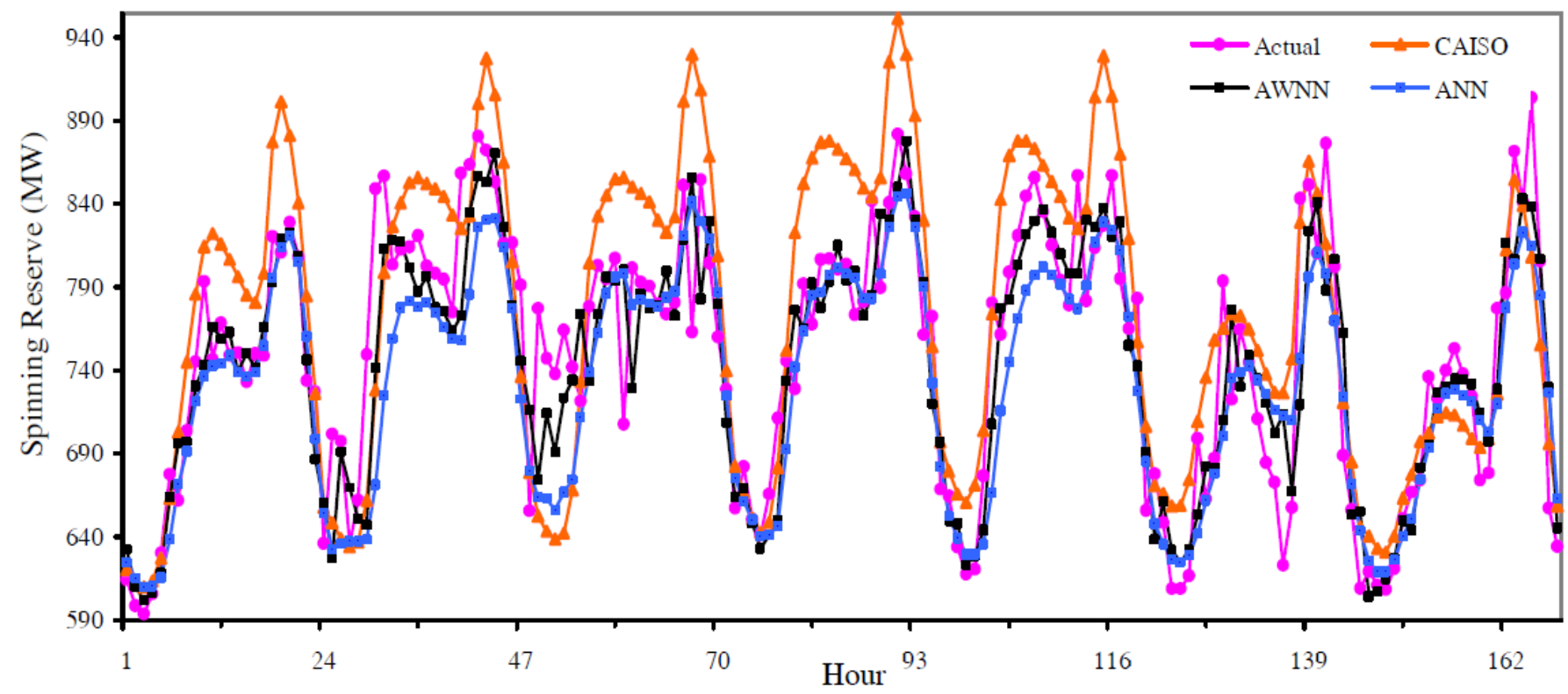

Fig. 5. Forecasting results for test week obtained by 20 . 


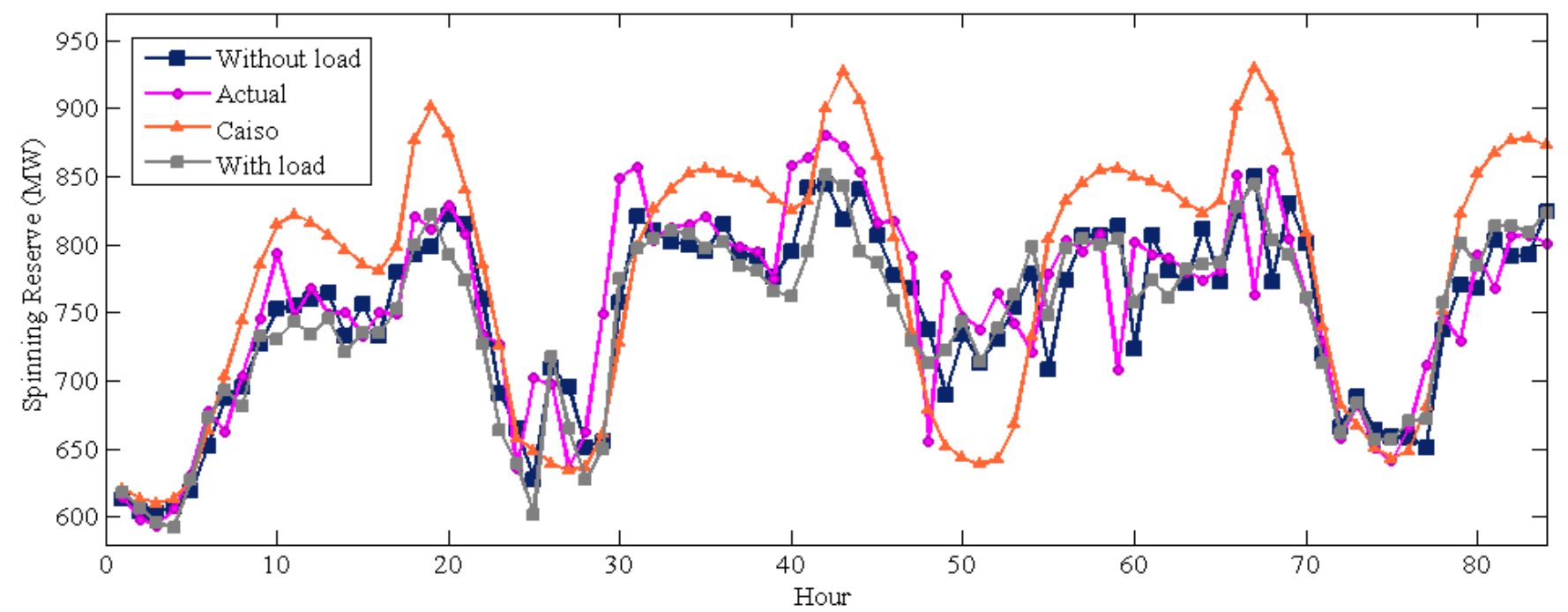

Fig. 6. Forecasting results for test week obtained by the proposed methodology and 20 (from 0 to 84 hour).

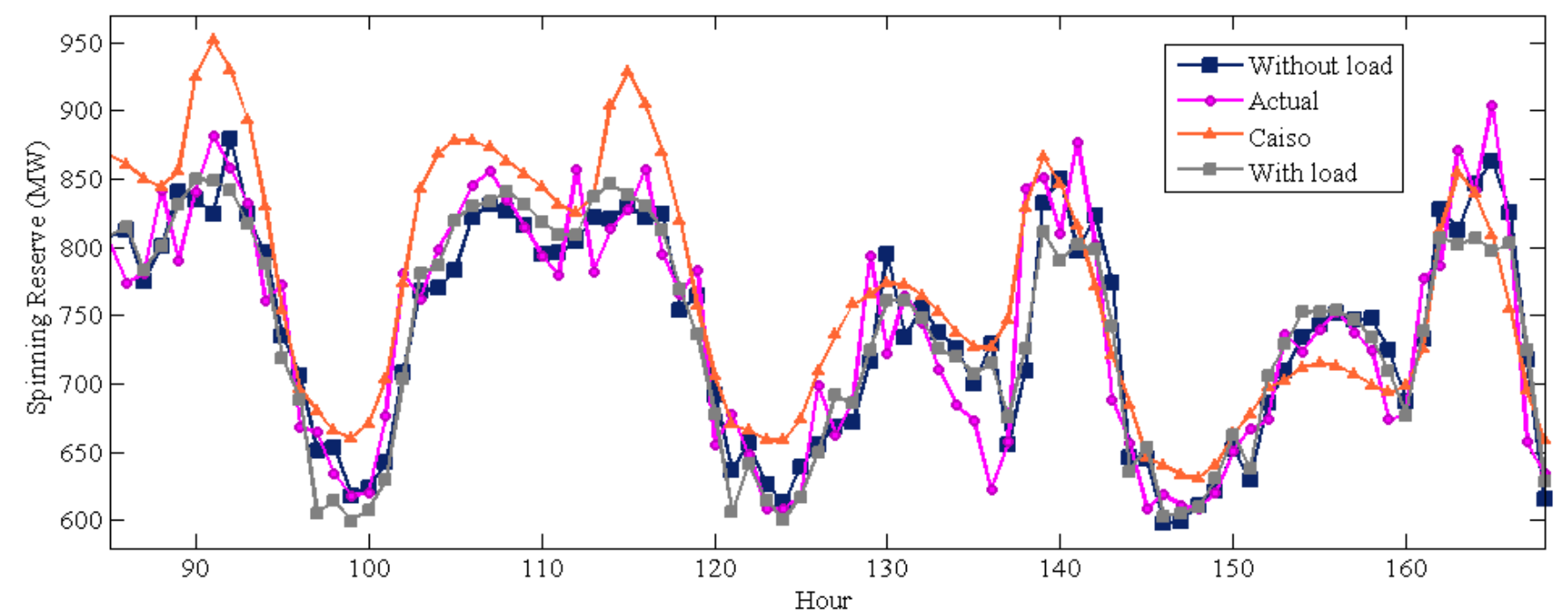

Fig. 7. Forecasting results for test week obtained by the proposed methodology and 20 (from 85 to 168 hour).

\section{REFERENCES}

[1] Z. Vale, C. Ramos, P. Faria, "Ancillary Service Market Simulation", IEEE T\&D Asia, Seoul, Korea, October 2009 (Accepted for publication)

[2] I. Praça, C. Ramos, Z. Vale, M. Cordeiro, "MASCEM: A Multi-Agent System that Simulates Competitive Electricity Markets", IEEE Intelligent Systems, vol. 18, no. 6, pp. 54-60, Special Issue on Agents and Markets, Nov-Dec 2003

[3] T. Pinto, Z. Vale, H. Morais, I. Praça, C. Ramos, "Multi-Agent Based Electricity Market Simulator With VPP: Conceptual and Implementation Issues", 2009 IEEE PES General Meeting, Calgary, Canada, July 2009

[4] F. Azevedo, Z. Vale, P. Oliveira, "A Decision-Support System Based on Particle Swarm Optimization for Multiperiod Hedging in Electricity Markets", IEEE Transactions on Power Systems, vol. 22, Issue 3, pp. 995-1003, Aug. 2007

[5] Z. Vale, H. Morais, M. Cardoso, C. Ramos, H. Khodr, "Distributed Generation Producers' Reserve Management", IEEE PES General Meeting 2008, Pittsburgh, USA, July 2008

[6] J. Taylor," "Neural Networks and Their Applications", John Wiley \& Sons, Ltd. (UK), 1996

[7] M. Hagan, H. Demuth, M. Beale, "Neural Network Design”, 1996
[8] CIGRE Task Force 38.05.07, "Methods and Tools for Costing Ancillary Services", CIGRE Publication, 2001

[9] R. Raineri, S. Ríos, D. Schiele, "Technical and economic aspects of ancillary services markets in the electric power industry: an international comparison", Energy Policy, vol. 34, Issue 13, pp. 15401555, September 2006

[10] L. Yonggang, J. Jiang, "Experience with Operating the AncillaryService Markets in ERCOT", IEEE Power Engineering Society General Meeting, 2007, pp. 1-6, 24-28 June 2007

[11] E. Amundsen, L. Bergman, "Provision of operating reserve capacity: Principles and practices on the Nordic Electricity Market", in Competition and Regulation in Network Industries, intersentia, 2007

[12] E. Miguélez, I. Cortésa, L. Rodríguez, G. Camino, "An overview of ancillary services in Spain”, Electric Power Systems Research, vol. 78, Issue 3, pp. 515-523, March 2008

[13] N. Pindoriya, S. Singh, S. Singh, "Forecasting the day-ahead Spinning Reserve requirement in competitive electricity market," IEEE Power and Energy Society General Meeting - Conversion and Delivery of Electrical Energy in the 21st Century,, July 2008

[14] http://oasis.caiso.com 